\title{
Real-time RSS-based positioning system using neural network algorithm
}

\author{
Safae El Abkari, Jamal El Mhamdi, El Hassan El Abkari \\ Electronic Systems, Sensors and Nanotechnologies Laboratory (E2SN), ENSAM, ST2I, \\ Mohammed V University in Rabat, Rabat, Morocco
}

\begin{tabular}{|c|c|}
\hline Article Info & ABSTRACT \\
\hline Article history: & Locating services have come under the spotlight in recent years in various \\
\hline Received Jun 13, 2020 & have low accuracy due to signal fluctuations. For this purpose, we present a \\
\hline Revised Jun 4, 2021 & Wi-Fi based locating system using artificial neural network to enhance the \\
\hline Accepted Jun 13, 2021 & $\begin{array}{l}\text { positioning process performances. We optimized the Levenberg Marquardt } \\
\text { algorithm to propose the better configuration of the multi-layer time-delay }\end{array}$ \\
\hline Keywords: & $\begin{array}{l}\text { perception neural network. We achieved an average error of } 10.3 \text { centimeters } \\
\text { with a grid of } 0.4 \text { meter in four tests. Yet, due to the instability of the }\end{array}$ \\
\hline $\begin{array}{l}\text { Multi-layer perception } \\
\text { Neural network }\end{array}$ & $\begin{array}{l}\text { received signal strength RSS-based locating systems present a limitation in } \\
\text { the resolution finesse that depends on the grid size. }\end{array}$ \\
\hline
\end{tabular}

Position

Positioning system

RSS

Wi-Fi

Wireless sensor network

\section{Corresponding Author:}

Safae El Abkari

Electronic Systems, Sensors and Nanotechnologies Laboratory (E2SN), ENSAM

ST2I, Mohammed V University in Rabat,

Avenue of United Nations, Agdal, Rabat, Morocco

Email: safae.abkari@um5s.net.ma
This is an open access article under the CC BY-SA license.

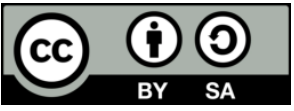

\section{INTRODUCTION}

Wireless technology has become popular because of its low-cost, wide communication range, mobility and computing capacities. Wireless communication refers to the use of radio signals to transmit data between two entities without any physical connection. There are various wireless technology types such as Bluetooth [1], ZigBee [2], and Wi-Fi [3]. Bluetooth uses IEEE802.15.1 standard which is very adaptable to personal area network applications. ZigBee uses IEEE802.15.4 standard, has low energy consumption but works on low data rate. Wi-Fi uses IEEE802.11 (a/b/n), has popular devices, works on high data rate but presents relatively high-power consumption. Table 1 shows a comparison of Bluetooth, ZigBee and Wi-Fi technologies.

In the last decade, wireless sensor networks have increasingly been used in various applications such as smart houses, work monitoring in factories and healthcare environments. For example, the use of WSN permits to monitor patients with telemedicine or healthcare services in hospitals [4]-[7]. Wi-Fi then comes as a technology that can be employed in WSN applications. Its key features are the popularity of Wi-Fi devices, affordability, and flexibility of the network. Wi-Fi sensors not only monitor but can also be used to locate within an environment [8]. Thus, there are two main localization methods as shown in Table 2: 
- Ranged-based localization [9]-[11]: this algorithm usually uses the information field of the tag geometry to determine its position. For this purpose, locating nodes and tag communication help to determine relative positions of the tag using collected geometric information.

- Range-free localization [12]: it consists of the calculation of mobile tag positions by finding locating nodes in its proximity. For this reason, this algorithm is also known as connectivity-based or proximity-based algorithm. With sufficient number of locating nodes, an accurate and robust localization is possible.

Table 1. Comparison of wireless locating technologies

\begin{tabular}{|c|c|c|c|c|c|c|}
\hline Technology & Main applications & Characteristics & Battery Life (days & Range (m) & Number of nodes & Rate $(\mathrm{kb} / \mathrm{s})$ \\
\hline Bluetooth & Cable Replacement & $\begin{array}{ll}- & \text { Low-cost } \\
- & \text { Ease of use }\end{array}$ & $1-7$ & $1-10$ & 7 & $20-250$ \\
\hline ZigBee & Monitoring and Control & $\begin{array}{ll}\text { - } & \text { Stability } \\
\text { - } & \text { Low energy } \\
& \text { consumption } \\
\text { - } & \text { Low-cost }\end{array}$ & $100-1000$ & $1-100$ & $255-65 \mathrm{k}$ & 720 \\
\hline Wi-Fi & Internet & $\begin{array}{ll}\text { - } & \text { Very High speed } \\
\text { - } & \text { Wide Range }\end{array}$ & Hours & 100 & 32 & $11 \mathrm{Mbps}$ \\
\hline
\end{tabular}

Table 2. Positioning method

\begin{tabular}{lccccc}
\hline & Energy Consumption & Accuracy & Deployment ability & Cost & Hardware \\
\hline Range-based & Medium-High & $90 \%$ & Hard & High-cost & Additional hardware required \\
Free-free & Medium & $70-75 \%$ & Easy & Low-cost & Additional hardware is not required \\
\hline
\end{tabular}

In order to determine the distance between two nodes, we perform a locating algorithm. If the collected physical quantities are measured and quantified at the receiving node, we can estimate the sensor tag position. The most popular locating algorithms used for this purpose are trilateration, proximity [13] and fingerprinting. A summary of those locating algorithms is illustrated in Table 3. While proximity algorithm is unused for range-based localization, fingerprinting [14], [15] can be more effective than trilateration [16], [17] because of its insensibility to received signal strength fluctuations, multipath signals and shadow fadings.

Table 3. Positioning algorithm summary

\begin{tabular}{|c|c|c|c|}
\hline Positioning algorithm & Signal Properties & Cons & Pros \\
\hline Trilateration & TOA/TDOA & Complex expensive sensitive to noise & Expensive high accuracy \\
\hline Proximity & RSS & Complex expensive & High accuracy \\
\hline \multirow[t]{3}{*}{ Fingerprinting } & RSS & Complex & Low-cost high performance \\
\hline & & Time consuming & \\
\hline & & High accuracy & \\
\hline
\end{tabular}

The objective of our proposed method is to determine a target node position with a low error compared to existing static models. Our main contributions are summarized as follows: i) We investigate a localization method based on neural network using RSS signals, and evaluate its performance in terms of network size and localization accuracy; ii) We process collected RSS signals using an average filter to make RSS data reliable and stable; iii) We provide an improved radio propagation model (RSS digital signature); iv) We introduce a time-delay multi-layer neural network localization algorithm to increase the positioning accuracy and applicability in real indoor environment; and v) Additionally, we study the accuracy metric to evaluate the positioning process using average distance error and sum square error.

The rest of the paper is organized as follows. Section 2 provides a literature review on different wireless-based positioning systems. In section 3, our proposed positioning system is presented in details; we firstly describe how we built our Wi-Fi based positioning system, secondly, we present our positioning methodology, thirdly, we describe the process of training/testing dataset collection and the multi-layer neural network. Experimental process, RSS dataset construction, empirical RSS signature and positioning error analyses are discussed in section 4. Finally, section 5 concludes our paper.

\section{RELATED WORKS}

Localization in wireless sensor network [18] (WSN) represents an emerging area of research. Many practical systems are still being developed and conceived. Hence, new algorithms and techniques are proposed for multiple layers of sensor network. The majority of wireless sensor network systems estimate 
positions using collected data which is possible only if the mobile tag transmits, collects data and uses a locating algorithm. Table 4 presents a survey of wireless-based positioning systems. In this paper, we propose a design of a real-time positioning system based on Wi-Fi and artificial neural network to enhance the accuracy of estimated positions. Furthermore, our proposed system can easily be adapted and deployed in various applications (positioning of robots, monitoring).

Table 4. Wireless-based positioning systems

\begin{tabular}{|c|c|c|c|c|c|}
\hline System & $\begin{array}{l}\text { Microsoft RADAR } \\
{[19]}\end{array}$ & Horus [20] & $\begin{array}{c}\text { Robot-based } \\
{[21]}\end{array}$ & TOPAZ [22] & DIT [23] \\
\hline Technology & $\mathrm{Wi}-\mathrm{Fi}$ & Wi-Fi & $\mathrm{Wi}-\mathrm{Fi}$ & Bluetooth+Infrared & Wi-Fi \\
\hline $\begin{array}{l}\text { Locating } \\
\text { technique }\end{array}$ & RSS & RSS & RSS & RSS & RSS \\
\hline $\begin{array}{l}\text { Positioning } \\
\text { algorithm }\end{array}$ & K-Neural Network & $\begin{array}{l}\text { Probabilistic } \\
\text { method }\end{array}$ & $\begin{array}{l}\text { Bayesian } \\
\text { approach }\end{array}$ & Neural Network & $\begin{array}{l}\text { Neural Network (multi-layer perception } \\
\text { (MLP), support vector machines (SVM)) }\end{array}$ \\
\hline Complexity & Medium & Medium & Medium & High & Medium \\
\hline Precision & $\begin{array}{l}50 \% \text { in } 2.5 \mathrm{~m} \text { area } \\
90 \% \text { in } 5.9 \mathrm{~m} \text { area }\end{array}$ & $\begin{array}{l}90 \% \text { in } 2.1 \mathrm{~m} \\
\text { area }\end{array}$ & $\begin{array}{l}50 \% \text { in } \\
1.5 \mathrm{~m} \text { area }\end{array}$ & $95 \%$ in $2 \mathrm{~m}$ area & $\begin{array}{l}90 \% \text { in } 5.12 \mathrm{~m} \text { area (SVM) } \\
90 \% \text { in } 5.40 \mathrm{~m} \text { area (MLP) }\end{array}$ \\
\hline Robustness & Good & Good & Good & Poor & Good \\
\hline Cost & Low & Low & edium & Medium & Low \\
\hline
\end{tabular}

\section{OUR PROPOSED POSITIONING SYSTEM}

\subsection{Wi-Fi based positioning network}

Wi-Fi network operates at $2.4 \mathrm{GHz}$ frequency band which is the ISM band (Industrial, Scientific and Medical). Wi-Fi is recommended for controlling and monitoring applications. We used ESP8266 module which transmits at $+14 \mathrm{Bm}$ that is $25 \mathrm{~mW}$ (IEEE 802.11n TX power), has a receiver sensitivity [24] of $-72 \mathrm{dBm}(6$ to the power of $-8 \mathrm{~mW}$ ) and has a communication range of approximately $20-40$ meters for indoor environments. We can configure the ESP8266 Wi-Fi module as shown in Figure 1 by configuring it into three different groups: Sensor tag, anchors and coordinator.

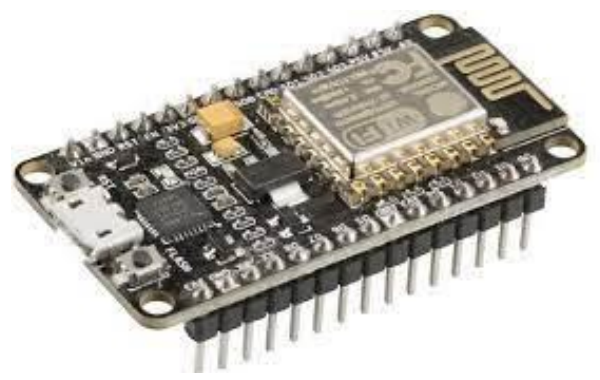

Figure 1. ESP8266 Wi-Fi module

\subsection{Positioning methodology}

Filtering raw RSS data using an average filter is an essential phase in developing a robust positioning model. Therefore, data partitioning phase starts after RSS data processing phase. This phase includes dividing processed RSS data into training and testing datasets. Training dataset is used to find and adjust the time-delay multi-layer perception (MLP) parameters in the offline phase. Testing dataset is used to evaluate and validate our positioning model.

\subsection{Collection of training/testing dataset}

We used the RSS-based method to estimate sensor tag location. received signal strength (RSS) is used to measure the incoming value from the target device when data have already been transmitted to anchors. We established the locating network by forming a mesh topology as shown in Figure 2, in order to identify and estimate positions of sensor tags within the Wi-Fi network. To request RSS values, the ESP8266 coordinator sends data to sensor tags at predetermined intervals as illustrated in Figure 3. 


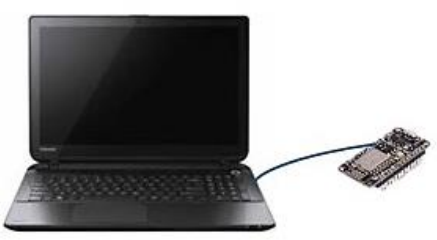

Coordinator

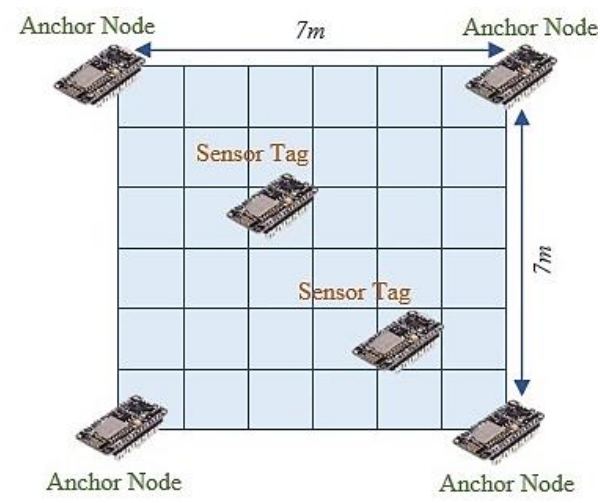

Anchor Node

Figure 2. Setup of Wi-Fi mesh network

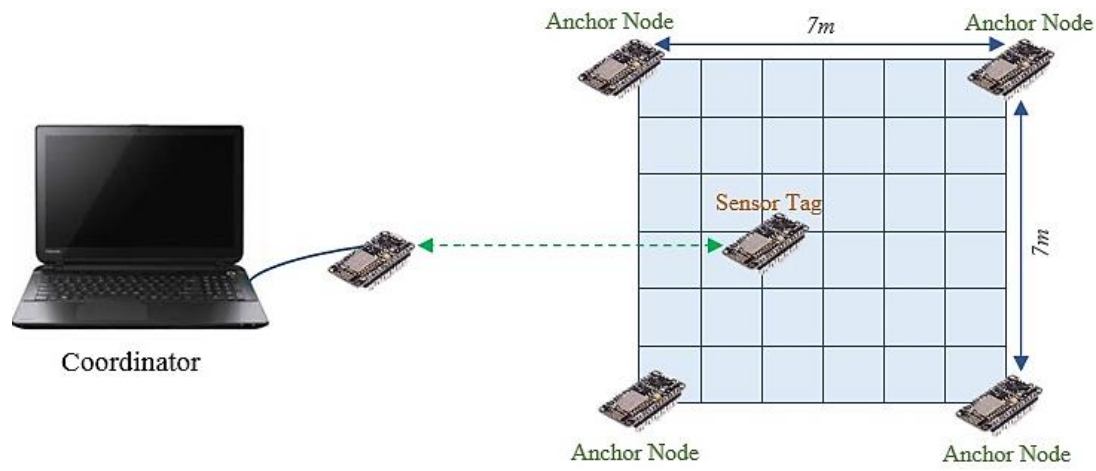

Figure 3. 1st step of RSS value request

Our system parts are: coordinator, sensor tags, and anchors. ESP8266 coordinator establishes a centralized personal area network and communicates with network anchors for routing. Positions will be determined at the coordinator level using RSS values when sensor tags are within the locating area. The process of RSS values' collection and acquisition is as follows: i) Initially, ESP8266 coordinator transmits data to sensor tags at predefined intervals to request RSS values; ii) Sensor tag Mac addresses (MAC) are already included into data sent from the coordinator. ESP8266 sensor tags transmit new data to anchors (Figure 4); and iii) When anchor nodes receive data from ESP8266 sensor tags, the anchors read RSS values, compare it to already stored RSS and extend RSS values into coordinator data format. Those data are then sent to coordinator to start positioning at the coordinator level (Figure 5).

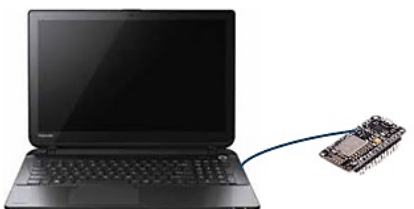

Coordinator

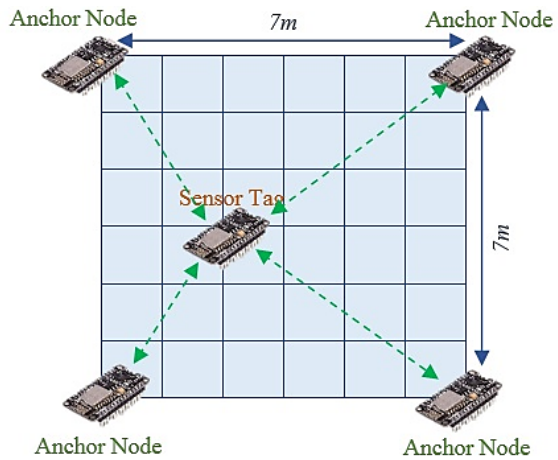

Figure 4. 2nd step of RSS value request 


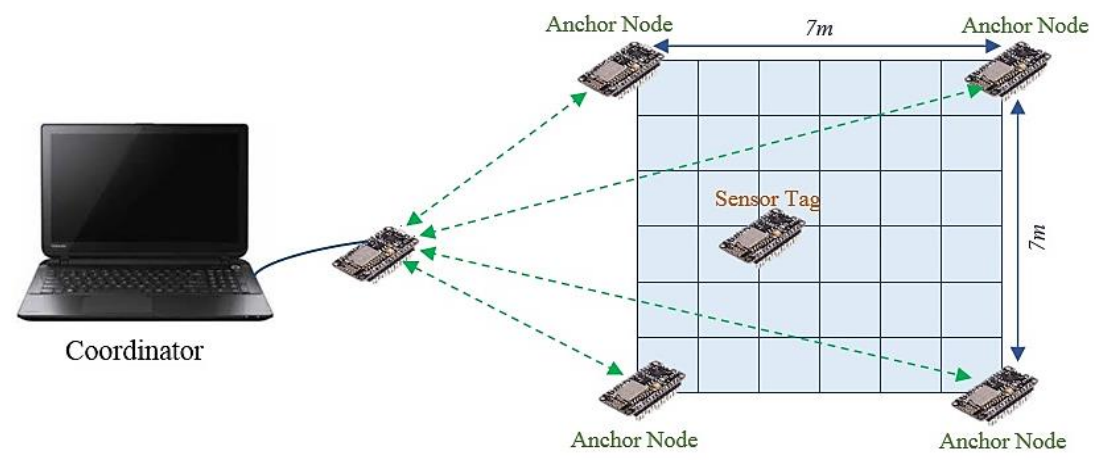

Figure 5. $3^{\text {rd }}$ step of RSS value request

\subsection{Multi-layer perception neural network}

Neural network is a mathematic model inspired from biological system. There is the multi-layer perception (MLP) which is a supervised learning technique that can be an alternative to implicit formula. We adapted the time-delay MLP to our positioning system to improve the system stability [25]-[27] because RSS values are not directly related to positions. The structure of time-delay MLP with two hidden layers is illustrated in Figure 6. Table 5 illustrates our proposed neural network schematic parameters.

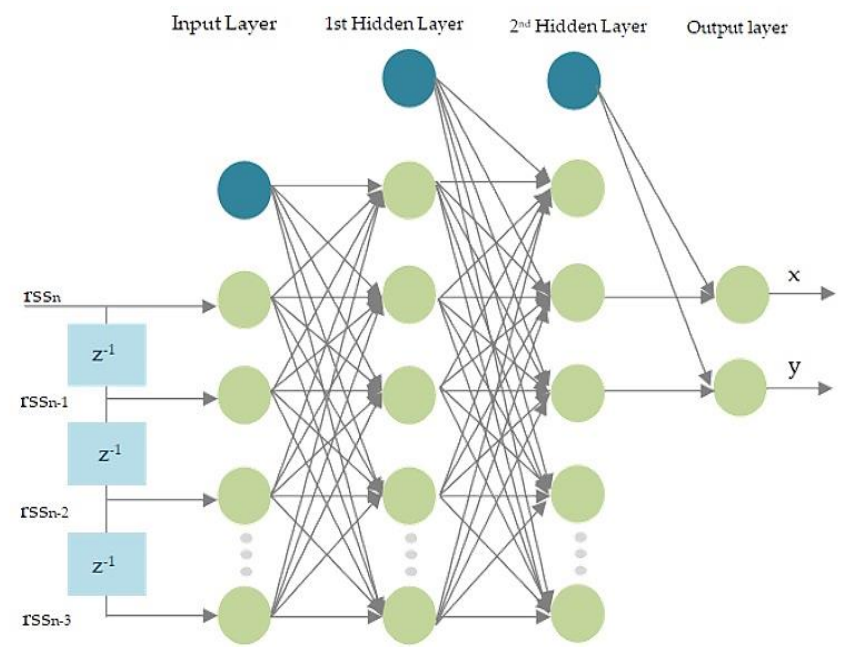

Figure 6. Time-delay multi-layer perception diagram

Table 5. The proposed neural network schematic

\begin{tabular}{ll}
\hline \multicolumn{1}{c}{ Input Type } & \multicolumn{1}{c}{ RSS of 4 anchor nodes } \\
\hline Input number & 24 \\
Time-delay number & 2 \\
Training algorithm & Levenberg-Marquardt \\
Hidden node size & $10-10-3$ \\
Activation Function of Hidden Nodes & Linear and hyperbolic-tangent sigmoid \\
Output number & 2 \\
Output type & $\mathrm{x}$ and y coordinates \\
\hline
\end{tabular}

Supervised neural network learning consists of:

- Phase 1: Training phase

Collection of data pattern to adjust (reduce) error of the interconnection weight of MLP using LevenbergMarquardt optimization (LM).

- Phase 2: Operation phase

Calculation of mobile tag positions using MLP (1), (2), (3).

$$
W_{i+1}=W_{i}-\left(J_{t}^{\bar{T}} J_{t}+\lambda I\right)^{-i} J_{t}^{T} J_{t}^{\bar{T}} E_{t}
$$




$$
\begin{aligned}
& d=b+\sum_{i=1}^{n} x_{i} w_{i} \\
& y=\frac{1}{1+e^{-d}}
\end{aligned}
$$

Where: wi+1, Wi is the weight of hidden layers; Jt is the output error derivative with respect to weight; Et is the error of output at $t$ time; $d$ is hidden node input; $b$ is the bias vector; $x i$ is data vector input; $y$ is the transfer function to activate output from hidden input node $\mathrm{d}$.

\section{EXPERIMENTAL RESULTS AND DISCUSSIONS}

For the experiment, we used an Intel Core i5 CPU, 8 GB of memory, Windows 8-pro 64-bit computer. ESP8266 modules were configured as anchor nodes, sensor tags and coordinator. We performed this experiment in an indoor room that contains tables, chairs, and computers. We firstly collected and processed raw RSS data to construst an RSS dataset. We studied the empirical RSS signature of our Wi-Fi network and evaluated the performances of the positioning using time-delay multi-layer perception neural network

\subsection{Construction of RSS dataset}

The first step of the experiment involves with the collection and the processing of raw RSS data. RSS signal can easily be affected by obstacles or orientations. Therefore, it is necessary to use a filter to smooth RSS signal and remove its fluctuations and sudden peaks. We used an average filter to determine average RSS values (4) of series of RSS signal values collected using anchors at a target position.

$$
R S S_{a v}=\frac{1}{n} \sum_{i=1}^{n} R S S_{i}
$$

In our study case, we calculated the average value of a serie of forthy RSS values collected at the same target position and at a defined interval. The filtred RSS signal values are stored in a database with its anchor position coordinates (Table 6).

$$
R S S_{a v}=\frac{1}{40} \sum_{i=1}^{40} R S S_{i}
$$

\begin{tabular}{|c|c|c|c|c|c|c|c|c|c|}
\hline \multirow{2}{*}{$\begin{array}{c}\text { Target position } \\
\text { coordinates }\end{array}$} & \multicolumn{3}{|c|}{ Anchor 1} & \multicolumn{3}{|c|}{ Anchor 2} & \multicolumn{3}{|c|}{ Anchor 3} \\
\hline & $\operatorname{Max}[\mathrm{dB}]$ & $\operatorname{Min}[\mathrm{dB}]$ & Mean [dB] & $\operatorname{Max}[\mathrm{dB}]$ & $\operatorname{Min}[\mathrm{dB}]$ & Mean $[\mathrm{dB}]$ & $\operatorname{Max}[\mathrm{dB}]$ & $\operatorname{Min}[\mathrm{dB}]$ & Mean $[\mathrm{dB}]$ \\
\hline$(0.5,2)$ & -49.25 & -31.87 & -42.10 & -51.42 & -46.23 & -47.89 & -78.36 & -59.33 & -62.74 \\
\hline$(1.7,3.1)$ & -54.76 & -37.64 & -45.75 & -63.18 & -61.44 & -62.28 & -57.61 & -52.47 & -54.88 \\
\hline$(7,4.5)$ & -21.75 & -16.04 & -19.15 & -37.66 & -24.75 & -32.47 & -62.77 & -53.64 & -57.12 \\
\hline
\end{tabular}

Table 6. Raw RSS data statistic for 40 RSS collection per anchor

\subsection{Empirical RSS signature of our Wi-Fi network}

The empirical RSS signature (Figure 7) is obtained by collecting average RSS signal values at predefined target points and their corresponding distances. Table 7 illustrates empirical RSS signature. Experimental tests showed that RSS values are relatively stable within nine meters. Therefore, we limited the testbed of our experiment within a $7 \times 7 \mathrm{~m}$ and a grid resolution of $0.4 \times 0.4 \mathrm{~m}$.

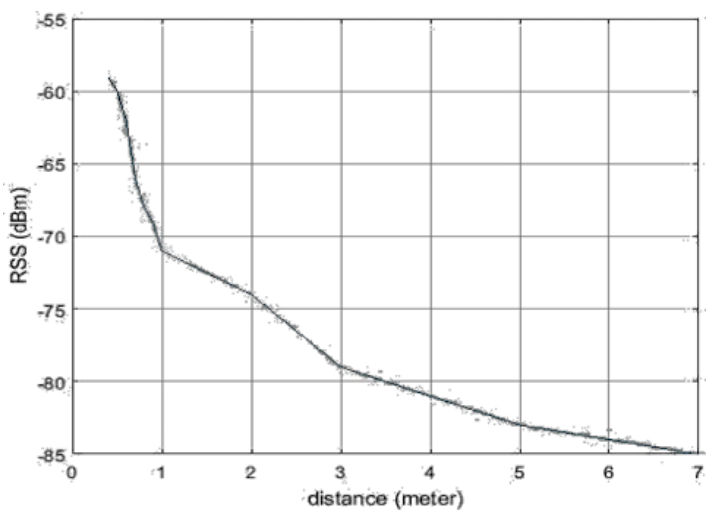

Figure 7. Empirical RSS signature 
Table 7. Signature of empirical RSS values

\begin{tabular}{cccc}
\hline Mean of RSS $(\mathrm{dBm})$ & Distance $(\mathrm{m})$ & Mean of RSS $(\mathrm{dBm})$ & Distance $(\mathrm{m})$ \\
\hline-59.56 & 0.4 & -72.98 & 2 \\
-59.84 & 0.5 & -77.66 & 3 \\
-61.65 & 0.6 & -80.97 & 4 \\
-66.15 & 0.7 & -82.85 & 5 \\
-68.45 & 0.8 & -83.32 & 6 \\
-69.27 & 0.9 & -84.32 & 7 \\
-71.36 & 1 & & \\
\hline
\end{tabular}

\subsection{Positioning using time-delay multi-layer perception neural network}

In this section, we demonstrate the accuracy of the time-delay MLP positioning process with the help of our experiment testbed. The scenario consists of $7 \times 7 \mathrm{~m}$ square area with four anchors placed at $(0,0),(7,0)$, $(0,7)$ and $(7,7)$ while the target node coordinates are those stored in the database with their corresponding RSS signal values. The positioning process using time-delay MLP algorithm consists of the two following phases:

- Phase 1: Training phase

In this phase, we set four vectors of average RSS values (training dataset) as inputs of the neural network and their (x, y) coordinates as outputs. Sensor tag is kept at the same position until RSS values are collected. We repeated the transmission of RSS vector for four times. We then trained the neural network using 10-10-3 structure of time-delay MLP. We qualified the neural network weight by the LevenbergMarquardt algorithm. We illustrate each test parameters in Table 8.

- Phase 2: Operation phase

We compared real coordinates (testing dataset) with estimated ones using time-delay MLP algorithm, and we kept parameters of the test with the best performance (Test 2). Results in Table 9 illustrate that the proposed positioning algorithm has a low error.

Table 8. The 10-10-3 time-delay MLP test parameter

\begin{tabular}{lccccc}
\hline & Epoch & Validation check & Gradient & Momentum & Performance \\
\hline Test 1 & 840 & 840 & $9.23 \times 10-12$ & $0.98 \times 10-11$ & $5.92 \times 10-3$ \\
Test 2 & 430 & 119 & $1.47 \times 10-12$ & $0.98 \times 10-11$ & $2.68 \times 10-8$ \\
Test 3 & 200 & 127 & $2.46 \times 10-12$ & $0.98 \times 10-10$ & $3.01 \times 10-5$ \\
Test 4 & 1200 & 980 & $1.36 \times 10-5$ & $0.98 \times 10-6$ & $5.54 \times 10-5$ \\
\hline
\end{tabular}

Table 9. Results of the best performance obtained in test 2

\begin{tabular}{|c|c|c|c|}
\hline \multicolumn{2}{|c|}{ Real coordinates } & \multicolumn{2}{|c|}{ Estimated coordinates } \\
\hline $\mathrm{x}$ & $\mathrm{y}$ & $\mathrm{X}$ & $\mathrm{Y}$ \\
\hline 0.4 & 0 & 0.399 & 0.0 \\
\hline 3 & 0 & 3.024 & 0.040 \\
\hline 7 & 0 & 7.0 & 0.023 \\
\hline 0.4 & 1 & 0.421 & 1.499 \\
\hline 3 & 1 & 3.004 & 1.108 \\
\hline 7 & 1 & 7.0 & 1.010 \\
\hline 0.4 & 2 & 0.354 & 2.009 \\
\hline 3 & 2 & 2.987 & 1.897 \\
\hline 7 & 2 & 7.021 & 2.133 \\
\hline 0.4 & 3 & 0.400 & 3.042 \\
\hline 3 & 3 & 3.100 & 3.0 \\
\hline 7 & 3 & 6.988 & 2.877 \\
\hline 0.4 & 4 & 0.420 & 4.0 \\
\hline 3 & 4 & 3.0 & 3.972 \\
\hline 7 & 4 & 7.079 & 4.120 \\
\hline 0.4 & 5 & 0.394 & 4.978 \\
\hline 3 & 5 & 3.065 & 5.022 \\
\hline 7 & 5 & 7.0 & 4.896 \\
\hline 0.4 & 6 & 0.403 & 6.0 \\
\hline 3 & 6 & 3.047 & 6.032 \\
\hline 7 & 6 & 6.889 & 6.074 \\
\hline 0.4 & 7 & 0.378 & 7.025 \\
\hline 3 & 7 & 3.103 & 7.217 \\
\hline 7 & 7 & 6.969 & 7.0 \\
\hline
\end{tabular}

We evaluated the accuracy of the locating process using average distance error (6), distance error (7) and sum square error (8). We show these results in Table 10. With the training and the testing processes, we 
obtained estimated and real positions that are approximately the same. Thus, accuracy of the locating changed in function of the grid size resolution.

$$
\begin{aligned}
& \bar{d}=\sum_{i=1}^{n} \frac{1}{n} d \\
& d=\sqrt{\left(x_{r, i}-x_{e, i}\right)^{2}+\left(y_{r, i}-y_{e, i}\right)^{2}} \\
& S S E i=\frac{1}{2}\left[\left(x_{r, i}-x_{e, i}\right)^{2}+\left(y_{r, i}-y_{e, i}\right)^{2}\right]
\end{aligned}
$$

In this study, we reached an accuracy average of $89 \%$ for a $0.4 \mathrm{~m}$ grid size. The resolution finesse decreased with the augmentation of expected coordinates' results due to the distinguishing difficulties of conflict points. Thus, ambient environment, ESP8266 module orientation, interferences and reflections represent the main factors that affect Wi-Fi signals. Therefore, it is necessary to recalibrate the system when initializing the positioning process. Ideally, in absence of obstacles and interferences, our system will work at its full performances.

Firdaus et al. [28] introduced signal strength values' adaptation based on user orientation to overcome its effect on the indoor positioning system (IPS) accuracy. Abkari [29] provided a compromise between feasibility and accuracy. They minimized RSS fluctuations using median filter and combined fingerprinting and trilateration approaches. Alomari [30] introduced a static path planning model for mobile anchor assisted in wireless sensor networks. Each node receives localization information and estimates its position with high accuracy. Xu [31] presented a 2-D/3-D locating algorithm using weighted centroid localization (WCL) technique which controls involved anchor nodes by an optimized threshold.

Wang [32] proposed a quadratic weighted centroid algorithm to improve accuracy and generalization of tunnel personnel systems. The range accuracy of received signal strength (RSS) was improved by gaussian filter and trilateration models, combining centroid algorithm and weighting factor. Abdelraouf [33] proposed a perpendicular distance-based approach (PD) utilizing received signal strength. This algorithm was theoretically analyzed and was compared to WCL algorithm and weighted-compensated weighted centroid localization algorithm (WC-WCL) by considering the accuracy parameter. The locating with four anchor nodes using neural network presented in [34] has an average error of 0.4855 meter for 2-D locating. Kawai [35] investigated MLP indoor positioning method using RSS. Kalman filter was used to process data to improve the accuracy. S. R Jondhale [36] proposed an RSS-based method using generalized regression neural network (GRNN) to obtain locations of a single moving target for $2 \mathrm{D}$ in a WSN. The proposition demonstrated a good tracking performance with an accuracy in the scale of centimeters. El Abkari [37] presented an RSS-based positioning system using Convolutional Neural Network. It reduced randomness and noise of collected RSS values. This approach was implemented in a single-floor and multi-grid layout. In our study, we used the time-delay multilayer neural network and obtained an average locating error of 10.3 centimeters for a $0.4 \mathrm{~m}$ grid size. Thus, our proposed algorithm is carried out in a real indoor environment and has a high accuracy whereas most litterature algorithms are simulation based. We show these results in Table 11.

Table 10. Average error and sum square error of our proposed system

\begin{tabular}{lccccc}
\hline \multicolumn{4}{c}{ average distance error $(\mathrm{m})$ sum } & square error $(\mathrm{m})$ & \multicolumn{3}{c}{ average distance error $(\mathrm{m})$ sum square error $(\mathrm{m})$} \\
\hline Test 1 & 0.109 & 2.678 & Test 3 & 0.011 & 0.042 \\
Test 2 & 0.027 & 0.314 & Test 4 & 0.104 & 3.016 \\
\hline
\end{tabular}

Table 11. Comparison of locating algorithms and our proposed method

\begin{tabular}{lllc}
\hline \multicolumn{1}{c}{ Locating algorithm } & \multicolumn{1}{c}{ Environment } & \multicolumn{1}{c}{ Average error } & Anchor number \\
\hline Radio Map [28] & Indoor & 3.5 & 3 \\
Fingerprinting+ Trilateration [29] & Indoor & 0.3 & 7 \\
Weighted Centroid [30] & Simulation & 1.009 & - \\
WCL(2-D) [31] & Simulation & $>3$ meters & 100 \\
WCBD [32] & Simulation & 0.8503 meter & 20 \\
& Simulation & 2.7363 meters (1st deployment) & 14 \\
WC-WCL [33] & & 5.3406 meters (2nd deployment) & 11 \\
Multi- layer Neural Network [34] & Simulation & 0.4855 meter & 4 \\
MLP - Fingerprinting [35] & Indoor & 1.75 meter & 4 \\
GRNN+ UKF [36] & Simulation & 0.3334 meter & - \\
CNN [37] & Indoor & 0.98 meter & 3 \\
Proposed technique & Indoor (real-time) $10.3 \mathrm{~cm}(0.4 \mathrm{~m}$ grid size) & 4 \\
\hline
\end{tabular}




\section{CONCLUSION}

In this paper, we presented a system that deals with the positioning of a person or an asset within a static environment. We implemented a real-time positioning algorithm based on the time-delay multilayer neural network and Wi-Fi technology. Results demonstrated that time-delay multi-layer is better than typical MLP neural network because we considered both actual and previous RSS vector values. In fact, we achieved an average error of 10.3 centimeters with a grid of 0.4 meter in four tests. In future works, we will adapt new algorithms to perform a positioning process with higher resolutions. We will extend our real-time positioning system by integrating more ESP8266 Wi-Fi modules to ameliorate the locating by identifying target tag orientation and direction.

\section{REFERENCES}

[1] A. K. M. M. Hossaina and W. Soh, "A Comprehensive Study of Bluetooth Signal Parameters for Localization," 18th International Symposium on Personal, Indoor and Mobile Radio Communications, Athens, 2007, pp. 1-5, doi: 10.1109/PIMRC.2007.4394215.

[2] Blumenthal, R. Grossmann, F. Golatowski, and D. Timmermann, "Weighted Centroid Localization in Zigbeebased Sensor Networks," International Symposium on Intelligent Signal Processing, Alcala de Henares, 2007, pp. 1-6, doi: 10.1109/WISP.2007.4447528.

[3] J. Biswas and M. Veloso, "WiFi localization and navigation for autonomous indoor mobile robots," International Conference on Robotics and Automation, 2010, pp. 4379-4384, doi: 10.1109/ROBOT.2010.5509842.

[4] H. Lee, K. Park, B. Lee, J. Choi, and R. Elmasri, "Issues in data fusion for healthcare monitoring," Proceedings of the 1st international conference on PErvasive Technologies Related to Assistive Environments, 2008, pp. 1-8, doi: 10.1145/1389586.1389590.

[5] S. D. Grigorescu, O. M. Ghita, C. K. Banica, and S. Potlog, A. M. Paraschiv, "Health monitoring solution using dedicated ZigBee sensor network," International Symposium on Advanced Topics in Electrical Engineering, 2013, doi: 10.1109/ATEE.2013.6563529.

[6] A. Redondi, M. Chirico, L. Borsani, M. Cesana, and M. Tagliasacchi, "An integrated system based on wireless sensor networks for patient monitoring, localization and tracking," Ad Hoc Networks, vol. 11, no. 1, pp. 39-53, 2013, doi: 10.1016/j.adhoc.2012.04.006.

[7] S. El Abkari, A. Jilbab, and J. El Mhamdi, "RFID Medication Management System in Hospitals," International Journal of Online and Biomedical Engineering, vol. 16, no. 12, pp. 155-168, 2020.

[8] O. Hernandez, V. Jain, S. Chakravarty, and P. Bhargava, "Position location monitoring using ieee 802.15. 4/zigbee ${ }^{\circledR}$ technology," Beyond bits, vol. 4, pp. 67-73, 2009.

[9] S. Pandey and V. Shirshu, "A range-based localization system in multihop wireless sensor networks: a distributed cooperative approach," Wireless Personal Communications, vol. 86, no. 2, pp. 615-634, 2016, doi: 10.1007/s11277-015-2948-3.

[10] F. Mekelleche and H. Haffaf, "Classification and comparison of range-based localization techniques in wireless sensor networks," Journal of Communications, vol. 12, no. 4, pp. 221-227, 2017, doi:10.12720/jcm.12.4.221-227.

[11] Thimmaiah, H. Sudha, and G. Mahadevan, "A range based localization error minimization technique for wireless sensor network," Indonesian Journal of Electrical Engineering and Computer Science (IJEECS), vol. 7, no. 2, pp. 395-398, 2017, doi:10.11591/ijeecs.v7.i2.pp395-403.

[12] E. Q. Shahra, T. R. Sheltami, and E. M. Shakshuki, "A comparative study of range-free and range-based localization protocols for wireless sensor network: Using cooja simulator," Sensor Technology: Concepts, Methodologies, Tools, and Applications, pp. 1522-1537, 2020, doi: 10.4018/978-1-7998-2454-1.ch071.

[13] S. T. Kouyoumdjieva and G. Karlsson, "Experimental Evaluation of Precision of a Proximity-based Indoor Positioning System," Annual Conference on Wireless On-demand Network Systems and Services (WONS), 2019, pp. 130-137, doi: 10.23919/WONS.2019.8795488.

[14] J. So, J. Y. Lee, C. H. Yoon, and H. Park, "An improved location estimation method for wifi fingerprint-based indoor localization," International Journal of Software Engineering and Its Applications, vol. 7, no.3 pp. 77-86, 2013.

[15] Shahra, T. R. Sheltami, and E. M. Shakshuki, "Comparative study of fingerprint and centroid localization protocol using cooja,” Procedia Computer Science, vol. 98, pp. 16-23, 2016, doi: 10.1016/j.procs.2016.09.006.

[16] O. S. Oguejiofor, A. N. Aniedu, H. C. Ejiofor, and A. U. Okolibe, "Trilateration based localization algorithm for wireless sensor network," International Journal of Science and Modern Engineering (IJISME), vol. 1, no. 10, pp. 2319-6386, 2013.

[17] X. Fang, L. Chen, "An Optimal Multi-Channel Trilateration Localization Algorithm by Radio-Multipath MultiObjective Evolution in RSS-Ranging-Based Wireless Sensor Networks," Sensors, vol. 20, no. 6, pp. 1798, 2020, doi: 10.3390/s20061798.

[18] N. Baccar, M. Jridi, and R. Bouallegue, "Adaptive Neuro-Fuzzy location indicator in wireless sensor networks," Wireless Personal Communications, vol. 97, no. 2, pp. 3165-3181, 2017, doi: 10.1007/s11277-017-4668-3.

[19] V. Bahl and V. Padmanabhan, "Enhancements to the RADAR user location and tracking system," Microsoft Research, 2000 
[20] M. A. Youssef, A. Agrawala, and A. U. Shankar, "WLAN location determination via clustering and probability distributions," International Conference on Pervasive Computing and Communications, 2003, pp. 143-150, doi: 10.1109/PERCOM.2003.1192736.

[21] Z. Xiang, S. Song, J. Chen, H. Wang, J. Huang, and X. Gao, "A wireless LAN-based indoor positioning technology," IBM Journal of research and development, vol. 48, no. 5.6, pp. 617-626, 2004, doi: $10.1147 / \mathrm{rd} .485 .0617$.

[22] B. Ottersten, M. Viberg, P. Stoica, and A. Nehorai, "Exact and large sample ML techniques for parameter estimation and detection in array processing," Radar Array Processing Simon Haykin, Ch. 4, 1993, doi: 10.1007/978-3-642-77347-1_4.

[23] M. Brunato and R. Battiti, "Statistical learning theory for location fingerprinting in wireless LANs," Computer Networks, vol. 47, no. 6, pp. 825-845, 2005, doi: 10.1016/j.comnet.2004.09.004.

[24] ESP8266 nodemcu module. [Online]. Available: https://michel.re/esp8266/

[25] S. Bhardwaj, "Ann for node localization in wireless sensor network," International Journal of Advanced Research in Electrical, Electronics and Instrumentation Engineering, vol. 2, no. 5, pp. 1724-1731, 2013.

[26] S. Kumar and L. Seong-Ro, "Localization with RSSI values for wireless sensor networks: An artificial neural network approach," International Electronic Conference on Sensors and Applications, vol. 1, 2014, doi:10.3390/ecsa-1-d007.

[27] S. El Abkari and J. Mhamdi. "The nervous system modeling algorithm for signal processing and communication in intelligent networks," International Conference on Advanced Technologies for Signal and Image Processing (ATSIP), 2016, pp. 703-709, doi: 10.1109/ATSIP.2016.7523182.

[28] Firdaus, N. A. Ahmad, and S. Sahibuddin, "Adapted WLAN Fingerprint Indoor Positioning System (IPS) Based on User Orientations," Recent Trends in Information and Communication Technology: Proceedings Conference of Reliable Information and Communication Technology, 2017, pp. 226-236, 2018, doi: 10.1007/978-3-319-59427-9_25.

[29] S. El Abkari, A. Jilbab, and J. El Mhamdi, "Wireless Indoor localization using fingerprinting and Trilateration," Journal of Advanced Research in Dynamical and Control Systems, vol. 12, no. 7, pp. 2597-2602, 2020, doi:10.5373/JARDCS/V12SP7/20202394.

[30] A. Alomari, F. Comeau, W. Phillips, and N. Aslam, "New path planning model for mobile anchor-assisted localization in wireless sensor networks," Wireless Networks, pp. 1-19, 2017, doi: 10.1007/s11276-017-1493-2.

[31] L. Xu, K. Wang, Y. Jiang, F. Yang, Y. Du, and Q. Li, "A study on 2D and 3D weighted centroid localization algorithm in wireless sensor networks," International Conference on Advanced Computer Control, 2011, pp. 155-159, doi: 10.1109/ICACC.2011.6016388.

[32] H. Wang, L. Liang, J. Xu, H. She, and W. Li, "A quadratic weighted centroid algorithm for tunnel personnel positioning," International Journal of Distributed Sensor Networks, vol. 16, no. 4, pp. 1550147720917021, 2020, doi: $10.1177 \% 2 \mathrm{~F} 1550147720917021$.

[33] A. Abdelraouf, M. Ashour, H. F. Hammad, and T. Elshabrawy, "The Study of Perpendicular Distance Approach Based on RSSI for Indoor Localization," International Conference on Innovative Trends in Computer Engineering, 2019, pp. 208-213, doi: 10.1109/ITCE.2019.8646597.

[34] M. Abdelhadi, M. Anan, and M. Ayyash, "Efficient artificial intelligent-based localization algorithm for wireless sensor networks," Cyber Journals: Multidisciplinary Journals in Science and Technology, Journal of Selected Areas in Telecommunications (JSAT), vol. 3, pp. 10-18, 2013.

[35] T. Kawai, K. Matsui, Y. Honda, G. Villarubia, and J. M. C. Rodriguez, "Preliminary study for improving accuracy on Indoor positioning method using compass and walking detect, Intelligent Systems and Computing, Jun. 2018, doi: 10.1007/978-3-319-62410-5_39.

[36] S. R Jondhale, R.S. Deshpande, "Kalman filtering framework-based real time target tracking in wireless sensor networks using generalized regression neural networks," IEEE Sens. J. .vol. 19, no.1, pp. 224-233, 2019, doi: 10.1109/JSEN.2018.2873357.

[37] S. El Abkari, A. Jilbab, and J. El Mhamdi, "RSS-based Indoor Positioning Using Convolutional Neural Network," International Journal of Online and Biomedical Engineering, vol. 16, no. 12, pp.82-93, 2020. 